\title{
Ableitung einer analytischen Darstellung der Epiellipside.
}

\author{
Von Otto Biermann in Brünn.
}

In den folgenden Zeilen soll die in der Maschinenlehre vorkommende Epiellipside kurz behandelt werden, die durch einen festen Punkt $A$ auf der Peripherie einer Ellipse $E^{\prime \prime}$ erzeugt wird, wenn diese ohne zu gleiten auf einer. festen, der ersten Ellipse ähnlichen Ellipse $E$ abrollt.

Die feste Ellipse $E$ mit den Halbachsen $a$ und $b(a>b)$ besitze gegenüber gewählten rechtwinkligen Coordinatenachsen die Gleichung

$$
x^{2} b^{2}+y^{2} a^{2}=a^{2} b^{2}
$$

die Ellipse $E^{\prime}$ mit den Halbachsen $m a$ und $m b$ habe eine solche Anfangslage, dass ihre Achsen den gleichnamigen Achsen von $E$ parallel laufen und der Punkt $(o, b)$ Berührungspunkt sei. - Es soll die Bahn dieses Punktes $A$ von $E^{\prime}$ verfolgt werden, wenn das Abrollen im Sinne der Uhrzeigerbewegung stattfindet.

Gelangt $A$ in dem Augenblicke, als der gemeinsame Berührungspunkt von $E$ und $E^{\prime}$ etwa $B^{\prime}\left(x^{\prime}, y^{\prime}\right)$ ist, nach $A^{\prime}$, so lässt sich die Lage von $A^{\prime}$ am einfachsten in Beziehung setzen zu der Stelle $B^{\prime}$, zu dem Winkel $\alpha^{\prime}$, um den man den positiven Theil der $y$ Achse im Sinne der Uhrzeigerbewegung um $O$ drehen muss, damit man eine Parallele $\mathrm{zu}$ der in $B^{i} \mathrm{zu}$ verzeichnenden Normalen $N^{\prime}$ an $E$ und $E^{\prime \prime}$ erhält, zu dem Winkel $\varphi^{\prime}$, um den man die kleine Achse von $E^{\prime}$ aus ihrer neuen Lage um den Schnittpunkt $C^{\prime}$ derselben mit $N^{\prime}$ im Sinne verkehrt der Uhrzeigerbewegung drehen muss, dass sie in die Normale $N^{\prime}$ fällt, und endlich zu der Entfernung $\overline{A^{\prime} C^{\prime}}$.

Nennt man die Coordinaten ron $C^{\prime}\left(x_{1}, y_{1}\right)$ und die Entfernung $\overline{A^{\prime} C^{\prime}} r$, so sind die Coordinaten von $A^{\prime}$ :

$$
\begin{aligned}
& x=x_{1}-r \sin \left(\alpha^{\prime}+\varphi^{\prime}\right), \\
& y=y_{1}-r \cos \left(\alpha^{\prime}+\varphi^{\prime} .\right.
\end{aligned}
$$


Die Winkel $\alpha^{\prime}$ und $\varphi^{\prime}$ stehen dadurch in Zusammenhang, dass der von der Stelle $(o, b)$ bis $\left(x^{\prime}, y^{\prime}\right)$ reichende Ellipsenbogen von $E$ gleich sein soll dem Ellipsenbogen $A^{\prime} B^{\prime}$ von $E^{\prime \prime}$.

Wenn man die feste Ellipse durch die Gleichungen

$$
x=a \sin \omega, y=b \cos \omega \quad(\omega=0 \ldots 2 \pi)
$$

darstellt und

$$
x^{\prime}=a \sin \omega^{\prime}, y=b \cos \omega^{\prime}
$$

ist, so wird der erste Ellipsenbogen von $E$ dureh das a fache des Integrales

$$
u=\int_{0}^{\omega^{\prime}} \sqrt{1-k^{2} \sin ^{2} \omega} d \omega
$$

dargestellt, wo $k^{2}=\frac{a^{2}-b^{2}}{a^{2}}$ ist. - Doch weil tg $\omega^{\prime}=\frac{x^{\prime} b}{y^{\prime} a}$ ist und die Tangente des Neigungswinkels $\left(\frac{\pi}{2}-\alpha^{\prime}\right)$ der Normale $N^{\prime}$ gegenüber der positiven Richtung der $x$ Achse gleich $\frac{a^{2} y^{\prime}}{b^{2} x^{\prime}}$ ist, wird

$$
\operatorname{tg} \omega^{\prime}=\frac{a}{b} \operatorname{tg} a^{\prime}=\operatorname{tg} a m u,
$$

und man weiß denselben Bogen darzustellen, wenn $\alpha^{\prime}$ gegeben ist. Die Ellipse $E$ hat die Parameterdarstellung:

$$
x=a^{2} \frac{\operatorname{tg} \alpha}{\sqrt{b^{2}+a^{2} \operatorname{tg}^{2} \alpha^{\prime}}}, y=b^{2} \frac{1}{\sqrt{b^{2}+a^{2} \operatorname{tg}^{2} \alpha^{\prime}}} .
$$

Für eine der ersten Ellipse ähnliche Ellipse $m^{2} b^{2} x^{2}+m^{2} a^{2} y^{2}=$ $=m^{4} a^{2} b^{2}$ mit den Halbachsen $m a$ und $m b$ ist der von einem Endpunkte des kleinsten Durchmessers bis zu einer Stelle $\bar{x}=m a \sin \bar{\omega}, \bar{y}=m b \sin \bar{\omega}$ verlaufende Bogen dureh

$$
m a \int_{0}^{\bar{\omega}} \sqrt{1-k^{2} \sin ^{2} \omega} d \omega
$$

ausgedrückt. Soll dieser Bogen dieselbe Länge haben wie der erste auf $E$, so muss man

$$
\bar{\omega}=a m \frac{u}{m}
$$

setzen; und dann ist

$$
\operatorname{tg} \varphi^{\prime}=\frac{m b}{m a} \operatorname{tg} \bar{\omega}=\frac{b}{a} \operatorname{tgam} \frac{u}{m} .
$$


Die weitere Aufgabe besteht nun darin, die Coordinaten $x_{1}, y_{1}$ und die Entfernung $r$ zu bestimmen. Doch das gelingt, wenn man die Gleichung der die feste Ellipse im Punkte $\left(x^{\prime}, y^{\prime}\right)$ von außen berührenden Ellipse mit den Halbachsen $m a$ und $m b$ angeben kann, deren kleine Achse gegenüber der Normalen $N^{\prime}$ in $\left(x^{\prime}, y^{\prime}\right)$ die Neigung $\varphi^{\prime}$ hat.

Die Gleichung der Ellipse $E^{\prime}$ in der neuen Lage ist von der Form

$$
a_{11} x^{2}+2 a_{12} x y+a_{22} y^{2}+2 a_{13} x+2 a_{23} y+a_{33}=0 ;
$$

und zunächst ist zu bemerken, dass diese Gleichung nur dann eine der Ellipse $E$ ähnliche Ellipse mit den Halbachsen $m a$ and $m b$ darstellt, wenn

$$
A_{33}=a_{11} a_{22}-a_{12}^{2}=m^{4} a^{2} b^{2} \text { und } a_{11}+a_{22}=m^{2}\left(a^{2}+b^{2}\right)
$$

ist, also

$$
\begin{gathered}
a_{11}-a_{22}= \pm \sqrt{m^{4}\left(a^{2}-b^{2}\right)^{2}+4 a_{12}^{2}} \\
a_{11}=\frac{1}{2}\left(m^{2}\left(a^{2}+b^{2}\right) \pm \sqrt{m^{4}\left(a^{2}-b^{2}\right)^{2}+4 a_{12}^{2}}\right) \\
a_{22}=\frac{1}{2}\left(m^{2}\left(a^{2}+b^{2}\right) \mp \sqrt{m^{4}\left(a^{2}-b^{2}\right)^{2}+4 a_{12}^{2}}\right)
\end{gathered}
$$

ist.

Zweitens sind die Coefficienten der Gleichung (a) so zu wählen, dass $\left(x^{\prime}, y^{\prime}\right)$ ein der Gleichung genügendes Wertesystem ist und dass die Tangente an die Curve $(a)$ in diesem Punkte mit der Tangente:

$$
b^{2} x x^{\prime}+a^{2} y y^{\prime}-a^{2} b^{2}=0
$$

übereinstimmt.

$\mathrm{Zu}$ diesem Zwecke muss

$$
\begin{aligned}
& a_{11} x^{\prime}+a_{12} y^{\prime}+a_{13}=-\lambda b^{2} x^{\prime} \\
& a_{12} x^{\prime}+a_{22} y^{\prime}+a_{23}=-\lambda x^{2} y^{\prime} \\
& a_{13} x^{\prime}+a_{23} y^{\prime}+a_{33}=\lambda a^{2} b^{2}
\end{aligned}
$$

sein, wo $\lambda$ einen Proportionalitätsfactor bedeutet; und nun ist die Gleichung jeder Curve II. Ordnung, welche die Ellipse $E$ in $\left(x^{\prime}, y^{\prime}\right)$ berührt, von der Form

$$
\begin{gathered}
a_{11} x^{2}+2 a_{12} x y+a_{22} y^{2}-2\left(\lambda b^{2} x^{\prime}+a_{11} x^{\prime}+a_{12} y^{\prime}\right) x- \\
-2\left(\lambda a^{2} y^{\prime}+a_{12} x^{\prime}+a_{22} y^{\prime}\right)+ \\
+\left(2 \lambda a^{2} b^{2}+a_{11} x^{\prime 2}+2 a_{12} x^{\prime} y^{\prime}+a_{22} y^{\prime 2}\right)=0 .
\end{gathered}
$$


Soll die Berührung von aufien stattfinden und die Curve $(\alpha)$ ihre convexe Seite zur $x$ Achse kehren, so muss

$$
\left(\frac{d^{2} y}{d x^{2}}\right)_{x^{\prime} y^{\prime}}=\frac{a_{11}\left(a^{2} y^{\prime}\right)^{2}-a_{12}\left(b^{2} x^{\prime}\right)\left(a^{2} y^{\prime}\right)+a_{22}\left(b^{2} x^{\prime}\right)^{2}}{\lambda\left(a^{2} y^{\prime}\right)^{3}}
$$

dasselbe Zeichen haben wie $y^{\prime}$, oder $\lambda$ muss dasselbe Zeichen wie der Zähler haben.

Da die Determinante der Gleichung ( $\beta$ ) lautet:

$$
\Delta=-\lambda^{2}\left[a_{11}\left(a^{2} y^{\prime}\right)^{2}-a_{12}\left(b^{2} x^{\prime}\right)\left(a^{2} y^{\prime}\right)+a_{22}\left(b^{2} x^{\prime}\right)^{2}\right],
$$

kann man schreiben:

$$
\left(\frac{d^{2} y}{d x^{2}}\right)_{x^{\prime} y^{\prime}}=-\frac{\Delta}{\left(a^{2} \lambda y^{\prime}\right)^{3}}
$$

Und beachtet man, dass die in der Gleichung $(\beta)$ enthaltene Curve, wenn sie auf ihre Achsen bezogen wird, durch eine der Gleichungen

$$
\begin{aligned}
& m^{2} b^{2} \xi^{2}+m^{2} a^{2} \eta^{2}=m^{4} a^{2} b^{2} \\
& m^{2} a^{2} \xi^{2}+m^{2} b^{2} \eta^{2}=m^{4} a^{2} b^{2}
\end{aligned}
$$

darzustellen sein soll, so muss

$$
\frac{\Delta}{A_{33}}=-m^{4} a^{2} b^{2}
$$

sein und somit

$$
\lambda^{2}=\frac{\left(m^{4} a^{2} b^{2}\right)^{2}}{a_{11}\left(a^{2} y^{\prime}\right)^{2}-a_{12}\left(b^{2} x^{\prime}\right)\left(a^{2} y^{\prime}\right)+a_{22}\left(b^{2} x^{\prime}\right)^{2}} .
$$

Hier aber schließt man, dass der Nenner positiv sein muss, weil $\lambda^{2}>0$ ist; daher muss nach dem Früheren $\lambda$ selbst $>0$ sein und zwar:

$$
\lambda=\frac{m^{4} a^{2} b^{2}}{+\sqrt{a_{11}\left(a^{2} y^{\prime}\right)^{2}-a_{12}\left(b^{2} x^{\prime}\right)\left(a^{2} y^{\prime}\right)+a_{22}\left(b^{2} x^{\prime}\right)^{2}}} .
$$

Unterwirft man also die Coeflicienten der Gleichung ( $\beta$ ), welche, im Falle $\left(x^{\prime}, y^{\prime}\right)$ mit $(o, b)$ zusammentrifft, in die Gleichung $m^{2} b^{2} x^{2}+m^{2} a^{2} y^{2}-2 y m^{2} a^{2} b^{2}(m+1)^{2}+m^{2} a^{2} b^{2}(2 m+1)=0$ übergehen soll, den drei Bedingungen: 


$$
\begin{gathered}
2 a_{11}=m^{2}\left(a^{2}+b^{2}\right)-\sqrt{m^{4}\left(a^{2}-b^{2}\right)^{2}+4 a_{12}^{2}}, \\
2 a_{22}=m^{2}\left(a^{2}+b^{2}\right)+\sqrt{m^{4}\left(a^{2}-b^{2}\right)^{2}+4 a_{12}^{2}}, \\
\lambda^{-1}=\frac{1}{\sqrt{2} m^{4} a^{2} b^{2}}\left[m^{2}\left(a^{2}+b^{2}\right)\left(\left(a^{2} y^{\prime}\right)^{2}+\left(b^{2} x^{\prime}\right)^{2}\right)-2 a_{12}\left(a^{2} y^{\prime}\right)\left(b^{2} x^{\prime}\right)+\right. \\
\left.+\sqrt{m^{4}\left(a^{2}-b^{2}\right)^{2}+4 a_{12}^{2}}\left(-\left(a^{2} y^{\prime}\right)^{2}+\left(b^{2} x^{\prime}\right)^{2}\right)\right]^{\frac{1}{2}}
\end{gathered}
$$

so ist nur noch diejenige Bedingung aufzustellen, unter welcher die kleine Achse der durch die Gleichung ( $\beta$ ) dargestellten Ellipse $E^{\prime}$ mit der Normale:

$$
\eta-y^{\prime}=\frac{a^{2} y^{\prime}}{b^{2} x^{\prime}}\left(\xi-x^{\prime}\right)
$$

den Winkel $\varphi^{\prime}$ einschlieft. Nun sind die Tangenten der Neigungswinkel der Achsen unserer Ellipse durch die Gleichung

$$
a_{12} \operatorname{tg}^{2} \theta+\left(a_{11}-a_{22}\right) \operatorname{tg} \theta-a_{12}=0
$$

definiert; also

$$
\begin{gathered}
\operatorname{tg} \theta=\frac{-\left(a_{11}-a_{22}\right) \pm m^{2}\left(a^{2}-b^{2}\right)}{2 a_{12}}= \\
=\frac{+\sqrt{m^{4}\left(a^{2}-b^{2}\right)^{2}+4 a_{12}^{2}} \pm m^{2}\left(a^{2}-b^{2}\right)}{2 a_{12}} .
\end{gathered}
$$

Weil die Gleichung der Ellipse $E^{\prime}$ in der Anfangslage, wo für die kleine Achse $\operatorname{tg} \theta=\infty$ gilt, den Coefficienten $a_{12}=0$ hat, so gibt die Formel

$$
\operatorname{tg} \vartheta=\frac{\sqrt{m^{4}\left(a^{2}-b^{2}\right)^{2}+4 a_{12}^{2}}+m^{2}\left(a^{2}-b^{2}\right)}{2 a_{12}}
$$

die Tangente des Neigungswinkels der kleinen Achse; und es ist nun

$$
\begin{gathered}
\operatorname{tg} \varphi^{\prime}=\frac{\frac{a^{2} y^{\prime}}{b^{2} x^{\prime}}-\frac{1}{2 a_{12}}\left(m^{2}\left(a^{2}-b^{2}\right)+\sqrt{m^{4}\left(a^{2}-b^{2}\right)^{2}+4 a_{12}^{2}}\right)}{1+\frac{a^{2} y^{\prime}}{b^{2} x^{\prime}} \frac{1}{2 a_{12}}\left(m^{2}\left(a^{2}-b^{2}\right)+\sqrt{m^{4}\left(a^{2}-b^{2}\right)^{2}+4 a_{12}^{2}}\right.}= \\
=\operatorname{tg}\left(\left(\frac{\pi}{2}-a^{\prime}\right)-\vartheta\right)
\end{gathered}
$$


zu setzen, so dass die Gleichung, welche zur Bestimmung von $\alpha_{12}$ dient, nunmehr lautet:

$$
\begin{aligned}
& a_{12}\left[a_{12}\left\{\left(\operatorname{tg}^{2} \varphi^{\prime}-1\right)\left(\left(b^{2} x^{\prime}\right)^{2}-\left(a^{2} y^{\prime}\right)^{2}\right)-4\left(a^{2} y^{\prime}\right)\left(b^{2} x^{\prime}\right) \operatorname{tg} \varphi^{\prime}\right\}+\right. \\
& \left.+m^{2}\left(a^{2}-b^{2}\right)\left(\left(b^{2} x^{\prime}\right)+\left(a^{2} y^{\prime}\right) \operatorname{tg} \varphi^{\prime}\right)\left(\left(b^{2} y^{\prime}\right) \operatorname{tg} \varphi^{\prime}-\left(a^{2} y^{\prime}\right)\right)\right]=0 .
\end{aligned}
$$

Die Lösung $a_{12}=0$ ist nicht brauchbar, denn da würde ja die Ellipse $E^{\prime}$ aus der Anfangslage nicht gedreht werden; und man erhält

$$
\begin{gathered}
a_{12}=\frac{m^{2}\left(a^{2}-b^{2}\right)\left(\left(a^{2} y^{\prime}\right) \operatorname{tg} \varphi^{\prime}+\left(b^{2} x^{\prime}\right)\right)\left(\left(a^{2} y^{\prime}\right)-\left(b^{2} x^{\prime}\right) \operatorname{tg} \varphi^{\prime}\right)}{\left(\left(a^{2} y^{\prime}\right)-\left(b^{2} x^{\prime}\right) \operatorname{tg} \varphi^{\prime}\right)^{2}-\left(\left(a^{2} y^{\prime}\right) \operatorname{tg} \varphi^{\prime}+\left(b^{2} x^{\prime}\right)\right)^{2}}= \\
=\frac{m^{2}\left(a^{2}-b^{2}\right)}{2} \operatorname{tg} 2\left(a^{\prime}+\varphi^{\prime}\right) .
\end{gathered}
$$

Jetzt ist die Gleichung der Ellipse $E^{\prime}$ bei beliebiger Lage während der Bewegung bekannt und man hat zur Ermittlung der Coordinaten $\left(x_{1}, y_{1}\right)$ und der Entfernung $r$ nur mehr den Schnittpunkt der Geraden

$$
y-\eta=\operatorname{tg} \vartheta(x-\xi) \text { and } y-y^{\prime}=\frac{a^{2} y^{\prime}}{b^{2} x^{\prime}}\left(x-x^{\prime}\right)
$$

zu berechnen, wo $\xi$ und $\eta$ die Coordinaten des Centrums der Ellipse $E^{\prime}$ sind, und hat zu beachten, dass $r$ der Summe von $m b$ und der Entfernung der Punkte $(\xi, \eta)$ und $\left(x_{1}, y_{1}\right)$ gleichkommt.

Die Coordinaten $\xi$ und $\eta$ lauten:

$$
\begin{aligned}
& \xi=x^{\prime}-\frac{\lambda}{A_{33}}\left(a_{12}\left(a^{2} y^{\prime}\right)-a_{22}\left(b^{2} x^{\prime}\right)\right), \\
& \eta=y^{\prime}+\frac{\lambda}{A_{33}}\left(a_{11}\left(a^{2} y^{\prime}\right)-a_{12}\left(b^{2} x^{\prime}\right)\right) .
\end{aligned}
$$

Man hat also alle Mittel zur Berechnung der Coordinaten von $A^{\prime}$ als Functionen von $\omega^{\prime}$ oder von $\alpha^{\prime}$.

Es ergibt sich unmittelbar, dass

$$
\begin{gathered}
2 a_{11}=m^{2}\left(a^{2}+b^{2}\right)-\frac{m^{2}\left(a^{2}-b^{2}\right)}{\cos 2\left(a^{\prime}+\varphi^{\prime}\right)} \\
2 a_{12}=m^{2}\left(a^{2}-b^{2}\right) \operatorname{tg} 2\left(\alpha^{\prime}+\varphi^{\prime}\right) \\
2 a_{22}=m^{2}\left(a^{2}+b^{2}\right)+\frac{m^{2}\left(a^{2}-b^{2}\right)}{\cos 2\left(\alpha^{\prime}+\varphi^{\prime}\right)} \\
\frac{\lambda a^{2} y^{\prime}}{2 m^{3} a^{2} b^{2}}= \\
=\left\{\frac{2\left(a^{2}+b^{2}\right)}{\cos ^{2} a^{\prime}}-\frac{2\left(a^{2}-b^{2}\right)}{\cos 2\left(\alpha^{\prime}+\varphi^{\prime}\right)}\left(\sin 2\left(\alpha^{\prime}+\varphi^{\prime}\right)+2 \operatorname{tg} \alpha^{\prime} \operatorname{cotg} 2 \alpha^{\prime}\right)\right\}^{-\frac{1}{2}}
\end{gathered}
$$


ist, und man kann die Curve, welche das Centrum der rollenden Ellipse beschreibt, durch die Gleichungen definieren:

$$
\begin{aligned}
& \xi=x^{\prime}+m\left(\frac{\lambda a^{2} y^{\prime}}{2 m^{3} a^{2} b^{2}}\right)\left[\left(a^{2}+b^{2}\right) \operatorname{tg} \alpha^{\prime}-\left(a^{2}-b^{2}\right) \frac{\sin 2\left(\alpha^{\prime}+\varphi^{\prime}\right)-\operatorname{tg} \alpha^{\prime}}{\cos 2\left(\alpha^{\prime}+\varphi^{\prime}\right)}\right], \\
& \eta=y^{\prime}+m\left(\frac{\lambda a^{2} y^{\prime}}{2 m^{3} a^{2} b^{2}}\right)\left[\left(a^{2}+b^{2}\right)-\left(a^{2}-b^{2}\right) \frac{1+\sin 2\left(\alpha^{\prime}+\varphi^{\prime}\right) \operatorname{tg} \alpha^{\prime}}{\cos 2\left(\alpha^{\prime}+\varphi^{\prime}\right)}\right] .
\end{aligned}
$$

Die Tangente des Winkels $\theta$ kommt gleich

$$
\operatorname{cotg}\left(\alpha^{\prime}+\varphi^{\prime}\right)
$$

die Coordinaten des Punktes $C^{\prime}\left(x_{1}, y_{1}\right)$ genügen den Gleichungen:

$$
\begin{aligned}
& y_{1}-x_{1} \operatorname{cotg}\left(\alpha^{\prime}+\varphi^{\prime}\right)+\left(\xi \operatorname{cotg}\left(\alpha^{\prime}+\varphi^{\prime}\right)-\eta\right)=0, \\
& y_{1}-x_{1} \operatorname{cotg} a^{\prime}+\frac{a^{2}-b^{2}}{\sqrt{b^{2}+a^{2} \operatorname{tg}^{2} \alpha^{\prime}}}=0,
\end{aligned}
$$

daher ist

und

$$
\begin{gathered}
x_{1}=\xi+\frac{\left(\xi \operatorname{cotg} \alpha^{\prime}-\eta_{1}\right)-\frac{a^{2}-b^{2}}{\sqrt{b^{2}+a^{2} \operatorname{tg}^{2} \alpha^{\prime}}}}{\operatorname{cotg}\left(\alpha^{\prime}+\varphi^{\prime}\right)-\operatorname{cotg} \alpha^{\prime}}, \\
y_{1}=\eta+\left(x_{1}-\xi\right) \operatorname{cotg}\left(\alpha^{\prime}+\varphi^{\prime}\right)
\end{gathered}
$$

$$
r=m b+\frac{x_{1}-\xi}{\sin \left(\alpha^{\prime}+\varphi^{\prime}\right)}
$$

Weil aber

$$
\begin{gathered}
\operatorname{cotg}\left(\alpha^{\prime}+\varphi^{\prime}\right)-\operatorname{cotg} \alpha^{\prime}=-\frac{\sin \varphi^{\prime}}{\sin \left(\alpha^{\prime}+\varphi^{\prime}\right) \sin \alpha^{\prime}}, \\
\operatorname{cotg} \alpha^{\prime}-\operatorname{tg} \alpha^{\prime}=2 \operatorname{cotg} 2 \alpha^{\prime}, \\
\xi \operatorname{cotg} \alpha^{\prime}-\eta-\frac{a^{2}-b^{2}}{\sqrt{b^{2}+a^{2} \operatorname{tg}^{2} \alpha^{\prime}}}= \\
=\left(\frac{\lambda a^{2} y^{\prime}}{\left.2 m^{3} \frac{a^{2} b^{2}}{2}\right)}\right) \frac{2 m\left(a^{2}-b^{2}\right)\left(1-\sin 2\left(\alpha^{\prime}+\varphi^{\prime}\right) \cdot \operatorname{cotg} 2 \alpha^{\prime}\right)}{\cos 2\left(\alpha^{\prime}+\varphi^{\prime}\right)}
\end{gathered}
$$

ist, wird

$$
r=m b-
$$

$$
2 m\left(\alpha^{2}-b^{2}\right)\left(1-\sin 2\left(\alpha^{r}+\varphi^{\prime}\right) \operatorname{cotg} 2 \alpha^{\prime}\right) \sin \alpha^{\prime}
$$

$\sin \varphi^{\prime} \cos 2\left(\alpha^{\prime}+\varphi^{\prime}\right) \sqrt{\frac{2\left(a^{2}+b^{2}\right)}{\cos ^{2} \alpha^{\prime}}-\frac{2\left(\alpha^{2}-b^{2}\right)}{\cos 2\left(\alpha^{\prime}+\varphi^{\prime}\right)}\left(\sin 2\left(\alpha^{\prime}+\varphi^{\prime}\right)+2 \operatorname{tg} \alpha^{\prime} \cdot \operatorname{cotg} 2 \alpha^{\prime}\right)}$. 
Jetzt sind alle Größen in den Ausdrücken für die Coordinaten eines beliebigen Punktes der einfachen Epiellipside als Functionen von $\alpha^{\prime}$ dargestellt, denn für $\varphi^{\prime}$ gilt die Beziehung

$$
\operatorname{tg} \varphi^{\prime}=\frac{b}{a} \operatorname{tgam} \frac{u}{m}
$$

wobei

$$
\frac{b}{a} \operatorname{tgam} u=\operatorname{tg} \alpha^{\prime}
$$

ist.

Wenn die Ellipse $E^{\prime}$ der festen Ellipse $E$ congruent ist, hat man $m=1$ und $\alpha^{\prime}=\varphi^{\prime}$ zu setzen, doch die Formeln für die Coordinaten $(x, y)$ der hier auftretenden einfachsten Epiellipside:

$$
x=\frac{a^{2} \operatorname{tg} \alpha^{\prime}}{\sqrt{b^{2}+a^{2} \operatorname{tg}^{2} \alpha^{\prime}}}-b \sin 2 \alpha^{\prime}+
$$

$+\left\{\left(a^{2}+b^{2}\right) \cdot \operatorname{tg} \alpha^{\prime} \cos 4 \alpha^{\prime}-\left(a^{2}-b^{2}\right)\left(\sin 4 \alpha^{\prime}-\operatorname{tg} \alpha^{\prime}+2 \cos 4 \alpha^{\prime}\left(\cos \alpha^{\prime}+\sin \alpha^{\prime}\right)^{2}\right)\right\} \frac{1}{N}$,

$$
y=\frac{b^{2}}{\sqrt{b^{2}+a^{2} \operatorname{tg}^{2} a^{\prime}}}-b \cos 2 a^{\prime}+
$$

$+\left\{\left(a^{2}+b^{2}\right) \cos 4 \alpha^{\prime}-\left(a^{2}-b^{2}\right)\left(\sin 4 \alpha^{\prime} \cdot \operatorname{tg} \alpha^{\prime}+1+2 \cos 4 \alpha^{\prime}\left(\cos \alpha^{\prime}+\sin \alpha^{\prime}\right)^{2} \operatorname{cotg} 2 \alpha^{\prime}\right)\right\} \frac{1}{N}$,

wo $N$ das Zeichen für den Ausdruck:

$\cos 4 \alpha^{\prime} \sqrt{\frac{2\left(a^{2}+b^{2}\right)}{\cos ^{2} \alpha^{\prime}}-\frac{2\left(a^{2}-b^{2}\right)}{\cos 4 \alpha^{\prime}}\left(\sin 4 \alpha^{\prime}+2 \operatorname{tg} \alpha^{\prime} \cdot \operatorname{cotg} 2 \alpha^{\prime}\right)}$

ist, sind noch sehr verwickelt, aber sie reichen natürlich hin, die Lage von $A^{\prime}$ für einen vorgelegten Wert von $\alpha^{\prime}$ zu berechnen.

Setzt man hier $a=b=r$, so gehen die Gleichungen der Cardioide hervor:

$$
\begin{aligned}
& x=r\left(2 \sin \alpha^{\prime}-\sin 2 \alpha^{\prime}\right), \\
& y=r\left(2 \cos \alpha^{\prime}-\cos 2 \alpha^{\prime}\right) .
\end{aligned}
$$

Wie die Rechnung auszuführen ist, wenn die Anfangslage von $E^{\prime}$ eine neue ist und die Bahn des Berührungspunktes von $E^{\prime}$ and $E$ aus dieser Lage verlangt wird, bedarf keiner Erläuterung. mehr. $\left.{ }^{1}\right)$

\section{März 1896.}

1) In dem VIII. Bd. des American Journal of Math. behandelte Lane die einfache Ellipside, wie ich kürzlich bemerkte. 